\title{
Carrier-envelope-offset dynamics and stabilization of femtosecond pulses
}

\section{Journal Article}

\section{Author(s):}

Helbing, F.W.; Steinmeyer, G.; Stenger, J.; Telle, H.R.; Keller, U.

Publication date:

2002

Permanent link:

https://doi.org/10.3929/ethz-b-000422497

\section{Rights / license:}

In Copyright - Non-Commercial Use Permitted

Originally published in:

Applied Physics B 74, https://doi.org/10.1007/s00340-002-0898-4 


\author{
F.W. HELBING ${ }^{1, \infty}$ \\ G. STEINMEYER ${ }^{1}$ \\ J. STENGER ${ }^{2}$ \\ H.R. TELLE ${ }^{2}$ \\ U. KELLER ${ }^{1}$
}

\section{Carrier-envelope-offset dynamics and stabilization of femtosecond pulses}

${ }^{1}$ Ultrafast Laser Physics Laboratory, Institute of Quantum Electronics, Swiss Federal Institute of Technology, ETH Hönggerberg - HPT, 8093 Zürich, Switzerland

${ }^{2}$ Physikalisch-Technische Bundesanstalt (PTB), 38116 Braunschweig, Germany

\section{Received: 20 September 2001/ \\ Revised version: 24 October 2001 \\ Published online: 27 June 2002 - () Springer-Verlag 2002}

ABSTRACT We analyze and stabilize fluctuations of the relative phase between the carrier and the envelope of a modelocked laser. Mechanisms generating fluctuations of the carrierenvelope-offset (CEO) phase are experimentally identified in lasers with and without prisms for dispersion compensation. One mechanism is amplitude-to-phase coupling via self-steepening. This mechanism translates power changes into variations of the CEO phase. A similar but much stronger effect is caused by beam-pointing variations in lasers with intracavity prisms. Both mechanisms convert power noise of the laser into phase noise and can be used to externally control or stabilize the CEO frequency by adjusting the pump power. Our measurements are well explained by a theoretical model. This investigation allowed us to obtain an unsurpassed stabilization of the CEO phase to $0.02 \mathrm{rad}$ rms for a frequency range from $0.01 \mathrm{~Hz}$ to $10 \mathrm{kHz}$. We extend the discussion to pulseamplification schemes and show that beam-pointing variations are also expected to have a strong influence on the CEO phase of amplified pulses. We discuss methods to reduce or avoid CEO noise by suitable design of the dispersion-compensation scheme, both in oscillators and in amplifiers.

PACS 42.65.Re; 42.62.Eh; 42.60.Mi

1

\section{Introduction}

Current state-of-the-art laser sources deliver optical pulses with a duration of about $5 \mathrm{fs}$, which corresponds to two optical cycles in the near infrared [1]. At this duration, the peak electric field strength of such a pulse shows a pronounced dependence on the relative phase between carrier and envelope of the pulse. In the following, we call this relative phase the carrier-envelope-offset (CEO) phase. For longer pulse durations, this previously inaccessible parameter does not play a role. For few-cycle pulses, however, a dependence of the conversion efficiency on the CEO phase $\varphi_{\text {CEO }}$ is obvious for processes like above-threshold ionization (ATI, [2]). In this process, the ionization probability shows a step-like behavior with electric field strength. Similarly, the strongly fluctuating photon numbers of high harmonics have been ex-

Fax: +41-1/633-1059, E-mail: helbing@iqe.phys.ethz.ch plained by a lack of control of the CEO phase [3]. Controlling the CEO phase is also an important prerequisite for several proposed attosecond generation schemes. A recent study on the duration of an isolated high-harmonic pulse revealed a pulse duration of about $1.5 \mathrm{fs}$ [4]. The absence of the anticipated attosecond time signature in these experiments was partly attributed to temporal averaging over different values of the CEO phase. These examples clearly identify $\varphi_{\text {CEO }}$ as an important parameter in a new regime of nonlinear optics. Because of the extremely short pulses required to see these effects, this regime has been termed extreme nonlinear optics [5]. At pulse durations of two cycles and below, the conversion efficiency starts to exhibit a noticeable dependence on the CEO phase. The higher the order of the nonlinear optical process, the more pronounced this effect is expected to be.

2

\section{Basic definitions}

The physical origin of the CEO phase is the difference between phase velocity $v_{\mathrm{p}}$ and group velocity $v_{\mathrm{g}}$ inside an oscillator cavity. If this cavity does not contain any dispersive elements, both velocities are equal and the position of a maximum of the electric field stays fixed relative to the maximum of the envelope. Intracavity dispersion, however, induces a per-roundtrip phase offset

$\Delta \varphi_{\mathrm{GPO}}=\frac{2 \pi}{\lambda} \int_{0}^{L}\left(n_{\mathrm{g}}(z)-n(z)\right) \mathrm{d} z=\frac{\omega^{2}}{c} \int_{0}^{L} \frac{\mathrm{d} n(z)}{\mathrm{d} \omega} \mathrm{d} z$

between the envelope and the carrier of an optical pulse. Here the coordinate $z$ is chosen along the propagation axis inside the cavity and $L$ is the effective length of the cavity, i.e. twice the geometrical length for a linear cavity. $n=c / v_{\mathrm{p}}$ is the refractive index, $n_{\mathrm{g}}=c / v_{\mathrm{g}}$ the group index, and $\omega$ the angular frequency. For a typical laser cavity [6], the combined groupphase offset (GPO) effect of the Ti:sapphire crystal, the air path, and the prism compressor can be estimated as about 250 optical cycles. If the carrier is advanced by an integer number of cycles relative to the envelope, the electric field structure exactly reproduces itself from pulse to pulse (compare Fig. 1). In the following, we therefore concentrate on the sub-cycle part of (1), which we define as the pulse-to-pulse CEO phase change

$\Delta \varphi_{\mathrm{CEO}}=\Delta \varphi_{\mathrm{GPO}} \bmod 2 \pi$. 


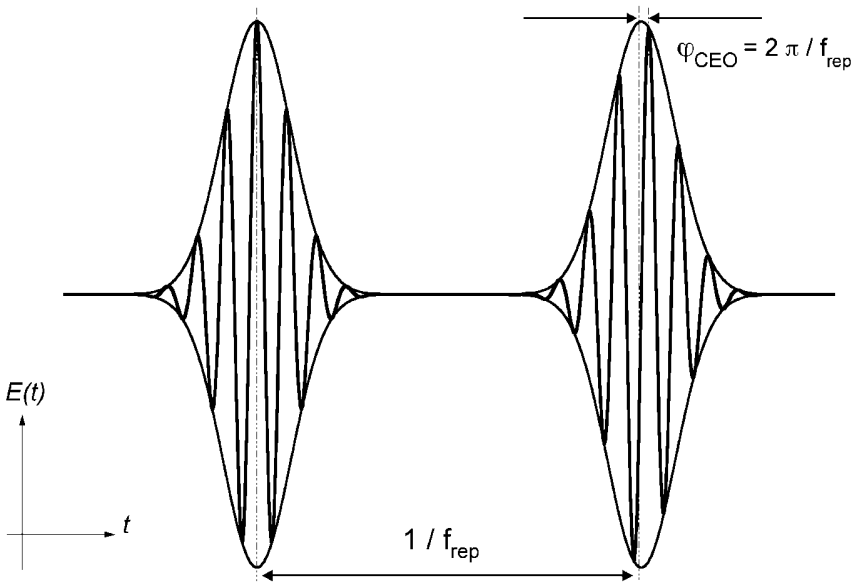

FIGURE 1 Electric field structure of pulses from a laser oscillator. The temporal delay between the envelope of two successive pulses is given by $1 / f_{\text {rep }}$, whereas the underlying carrier experiences an additional temporal shift caused by the difference of group and phase velocities in the laser cavity

For the case of a laser oscillator we define the CEO frequency as

$f_{\mathrm{CEO}}=\frac{1}{2 \pi} \frac{\mathrm{d} \varphi_{\mathrm{CEO}}}{\mathrm{d} t}=\frac{\Delta \varphi_{\mathrm{CEO}}}{2 \pi T_{\mathrm{R}}}=\frac{\Delta \varphi_{\mathrm{CEO}}}{2 \pi} f_{\mathrm{rep}}$.

It is important to note that any nonlinear or thermal change of the group-phase offset inside the laser cavity proportionally translates into a change of CEO frequency, whereas extracavity only the temporal derivative of such a GPO change may influence the CEO frequency. In other words, intracavity GPO always accumulates over many roundtrips, whereas extracavity GPO effects are only seen once in transmission. Given that noise is dominant at $\mathrm{kHz}$ frequencies corresponding to at least thousands of roundtrips, we will neglect GPO effects in the continuum-generation fiber in the following.

The first experimental approach to the measurement of the carrier-envelope offset relied on an interferometric cross correlation of two subsequent pulses [7]. However, even the slightest dispersive asymmetry between the two correlator arms introduces an offset to the measurement of $f_{\mathrm{CEO}}$, which makes this method inappropriate for any long-term stabilization of $\varphi_{\mathrm{CEO}}$.

An alternative approach to measurement of $f_{\mathrm{CEO}}$ that does not suffer from offset problems was first introduced in 1999 [8]. This method can be most suitably understood in the spectral domain (see Fig. 2). Fourier transforming the repetitive pulse train of Fig. 1 yields a comb of equidistant spectral lines $v_{m}=f_{\text {CEO }}+m f_{\text {rep }}$. Notably, this comb does not include zero frequency unless the pulse-to-pulse phase slip $\Delta \varphi_{\mathrm{CEO}}$ is exactly zero. The frequency comb must not be confused with the modes of the linear cavity, which are only equidistant in the absence of intracavity dispersion. For a mode-locked laser, the spacing of the comb lines is determined by the group velocity, i.e. the cavity roundtrip time of the envelope. Experimentally, the equidistance of these comb lines has been checked to a relative uncertainty better than $10^{-15}$ [9]. Any irregularity of the comb frequencies would automatically induce different repetition rates of the spectral components of the comb and eventually cause temporal spreading of the pulses. The fact that the comb only has two degrees of free-



FIGURE 2 Mode comb of a mode-locked laser oscillator in the frequency domain. The equidistantly spaced modes are represented by thick lines. Extending the comb towards zero frequency (thin lines) reveals an offset at zero frequency. This offset frequency can be measured by taking a frequency from the low-frequency wing of the spectrum, frequency-doubling it, and comparing it to a neighboring mode in the high-frequency wing of the spectrum

dom, $f_{\text {CEO }}$ and $f_{\text {rep}}$, has found widespread applications in metrology. Knowledge of these two frequencies provides one with a set of reference frequencies throughout the spectral coverage of the mode-locked laser. Beating an unknown optical frequency with the comb then allows one to derive the frequency of an optical transition from the measurement of three radio frequencies [10-12].

The picture in Fig. 2 also provides the key to measurement of $f_{\mathrm{CEO}}$ by heterodyning harmonics from different parts of the mode-locked spectrum [8]. Taking the $N$ th harmonic of a comb line $N v_{m_{1}}=N f_{\text {CEO }}+N m_{1} f_{\text {rep }}$ and beating it with the $M$ th harmonic of another comb line $M v_{m_{2}}=M f_{\mathrm{CEO}}+$ $M m_{2} f_{\text {rep }}$ yields

$M v_{m_{2}}-N v_{m_{1}}=(M-N) f_{\mathrm{CEO}}$

which requires $N m_{1}=M m_{2}$. This condition requires a certain minimum spectral width of the comb $\Delta f / f=2(N-$ $M) /(N+M)$; e.g. beating of the fundamental and the second harmonic requires an optical octave of bandwidth with $\Delta f / f=0.67$. This situation is illustrated in Fig. 2. Equation (4) is the key to any measurement of the carrier-envelope offset. This beat note delivers the carrier-envelope phase slippage rate, either directly or as one of its harmonics.

However, even the most broadband laser sources are hardpressed to directly deliver an optical octave. The requirement for spectral width can be alleviated by choosing higher harmonics, e.g. $N=2, M=3$. For oscillators with nJ energies, this can only slightly reduce the problem. Other methods based on optical transfer oscillators or interval bisection may be applied for much narrower spectra [8]. The simplest way to overcome bandwidth restrictions is the use of additional spectral broadening by self-phase modulation in optical fibers [10]. With the advent of microstructure fibers, whitelight continuum generation has become very simple even with the limited pulse energies of optical oscillators [13]. Spectral widths exceeding two optical octaves have been demonstrated in such fibers. The dominant effect behind the continuumgeneration process inside the fiber is self-phase modulation, which provides spectral broadening without affecting the average values of the frequency comb parameters $f_{\text {rep }}$ and $f_{\text {CEO }}$. On short time scales, deviations from the average values may well be apparent and cause fluctuations of the CEO 
phase. An analysis of this problem will be presented in Sect. 3. Other effects, such as Raman contributions to the fiber nonlinearity, may also be present and wash out the phase coherence between remote spectral components of the continuum. As long as these contributions can be kept low, it does not matter whether the spectral broadening takes place inside [14] or outside [10] the oscillator.

\section{Experimental characterization of carrier-envelope-offset noise of an oscillator}

To further investigate the behavior of the carrierenvelope-offset phase in Ti:sapphire lasers we set up the heterodyne detection scheme of Sect. 2; see Fig. 3. In most of the experiments, we employ a Ti:sapphire oscillator similar to the set-up described in [6]. This laser uses a set of two fused-silica prisms in combination with chirped mirrors for dispersion compensation and delivers 18 -fs pulses at $200-\mathrm{mW}$ output power. Given the output coupling of $2.5 \%$, this translates into an intracavity peak power of $4.5 \mathrm{MW}$ and an intensity of $4.5 \times 10^{11} \mathrm{~W} / \mathrm{cm}^{2}$ inside the Ti:sapphire crystal with a 2.3-mm path length. Later, this laser was converted to prismless dispersion compensation, which exclusively relies on chirped mirrors $[15,16]$. To allow for higher output powers, output coupling was increased to 5\%. Except for the mirrors used in the laser, pumping and mechanical set-up has been left widely unchanged to allow a direct comparison of the two lasers. The modified laser delivers 10 -fs pulses at $500-\mathrm{mW}$ output power. Focusing inside the crystal is unchanged. We therefore estimate an intracavity peak power of about $10 \mathrm{MW}$ and an intensity of $10^{12} \mathrm{~W} / \mathrm{cm}^{2}$.

For the heterodyne detection scheme, we additionally broaden the laser output spectrum by white-light continuum generation in a microstructure fiber, regardless of the laser used. We utilize the fundamental and the second harmonic $(N=1$ and $M=2$ in (4)). For frequency-doubling of the long-

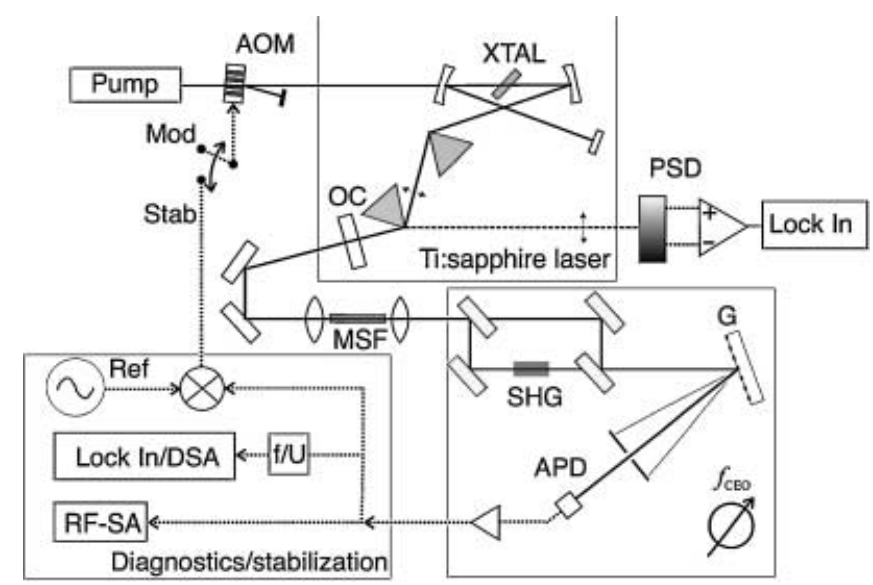

FIGURE 3 Experimental set-up. AOM: acousto-optic modulator. Mod: modulation frequency, used for amplitude modulation of pump light. XTAL: Ti:sapphire crystal. PSD: position-sensitive detector. OC: output coupler. MSF: microstructure fiber, used for continuum generation. SHG: $10-\mathrm{mm}$ LBO crystal, used for noncritically phase-matched second-harmonic generation of $1100 \mathrm{~nm}$. G: grating, used for spectral filtering. APD: avalanche photodiode. RF-SA: radio-frequency spectrum analyzer. f/U: frequency-tovoltage converter for FM demodulation. Lock In: lock-in amplifier. DSA: dynamical signal analyzer. Ref: reference oscillator used in the phase-lock wavelength components at $1060 \mathrm{~nm}$ a 1 -cm-long LBO crystal is used. Noncritical phase matching of second-harmonic generation (SHG) to yield $530 \mathrm{~nm}$ is achieved at a crystal temperature of $155^{\circ} \mathrm{C}$. Given the $1-\mathrm{cm}$ length of the crystal, one calculates a phase-matching bandwidth of about $4 \mathrm{~nm}$, accommodating about 40000 laser modes. Fundamental and SHG components at $530 \mathrm{~nm}$ are heterodyned in a Michelson interferometer. Careful optimization of the arm lengths in this interferometer is required for detection of the beat signal. Note that all modes within the phase-matching bandwidth contribute to the beat signal. Experimental parameters of the laser were set to ensure maximum intensity and stability of the continuum components at $1060 \mathrm{~nm}$ and at $530 \mathrm{~nm}$.

Figure 4 shows a typical rf spectrum of the signal detected by the avalanche photodiode in Fig. 3. The CEO beat notes are clearly visible at 35 and $65 \mathrm{MHz}$ at $45 \mathrm{dBc}$ in a $300-\mathrm{kHz}$ bandwidth. It needs to be pointed out that the quality of this signal is the most important prerequisite for any attempt to lock the carrier-envelope offset. In the unstabilized laser with prism dispersion compensation the frequency of this beat note may change very rapidly by up to several $\mathrm{MHz}$ in one second. This tendency is already greatly reduced by enclosing the laser in a box and carefully avoiding atmospheric turbulence. Excursions of the CEO frequency are further decreased by switching over to a prism-less laser set-up. With these improvements, the CEO frequency stays within a $500-\mathrm{kHz}$ interval for minutes of observation and without any active stabilization.

For a more thorough characterization of the fluctuations of $f_{\text {CEO }}$ we electronically convert the frequency into a proportional voltage. The control voltage of the phase-locked loop of the frequency-to-voltage converter is then analyzed by a dynamic signal analyzer (HP3562A). This allows one to de-

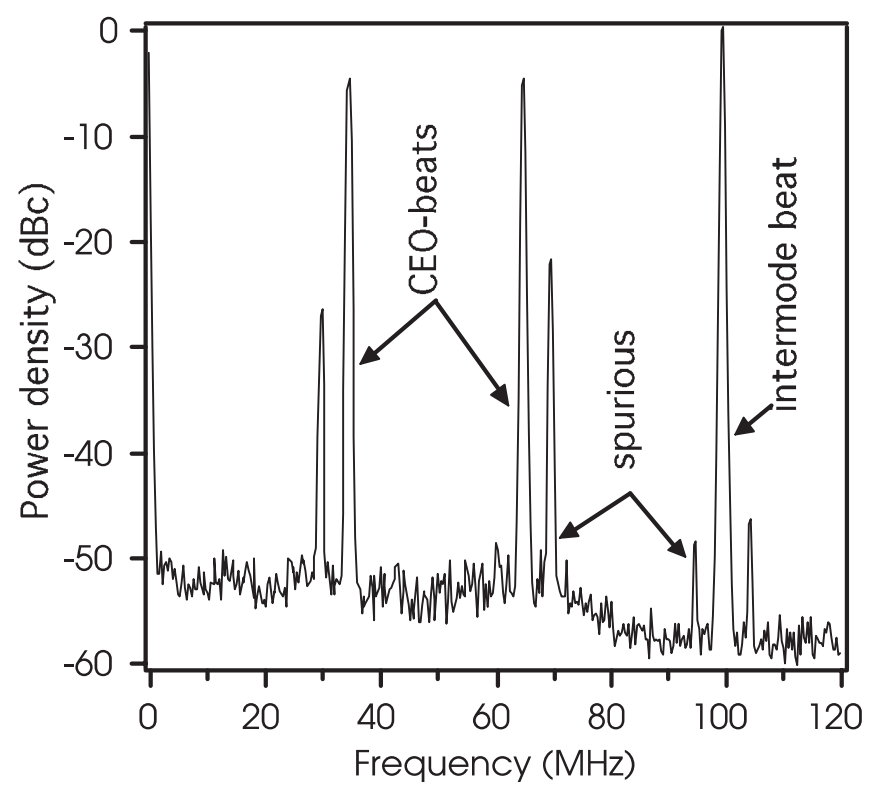

FIGURE 4 RF laser spectrum containing CEO beats at 35 and $65 \mathrm{MHz}$ as measured by the APD in Fig. 3. Resolution bandwidth was set to $300 \mathrm{kHz}$. The spectrum also shows some spurious components caused by intermodulation in the avalanche photodiode. These spurious components did not affect the measurements 
termine the single-sideband frequency noise density $\sigma_{f_{\mathrm{CEO}}}(f)$ in units of $\mathrm{Hz} / \sqrt{\mathrm{Hz}}$ as a function of offset frequency $f$. This is in full analogy to spectrally resolving amplitude noise and timing jitter in mode-locked lasers [17]. Figure 5 depicts measurements of the CEO noise of the unstabilized laser with intracavity prisms and the prism-less laser with and without stabilization. All measurements are composed of several sweeps with different spectral resolutions and are combined in a logarithmic plot, covering the range from $1 \mathrm{~Hz}$ to $100 \mathrm{kHz}$. The noise spectra typically show some discrete components at line-frequency harmonics and a broad background reaching up to several $\mathrm{kHz}$ offset frequency. The laser with intracavity prisms shows by far the worst noise behavior, with a pronounced maximum centered at about $500 \mathrm{~Hz}$. The prism-less laser shows a more than 10 times improved passive stability. A further reduction of the noise can be achieved with an active stabilization. This servo loop utilizes the dependence of the CEO frequency on intracavity peak power and adjusts this quantity by regulating the pump power with an acoustooptic modulator (AOM). The physical mechanisms behind this servo loop will become clear below. From the measured frequency noise density we calculate rms values using

$\delta f_{\text {CEO }}=\sqrt{2 \int_{f_{\text {low }}}^{f_{\text {high }}} \sigma_{f_{\text {CEO }}^{2}}^{2}(f) \mathrm{d} f .}$

Ideally, the integration should be extended up to the repetition rate of the laser. As can be seen in Fig. 5, the noise rolls off very rapidly at high frequencies, and the $100-\mathrm{kHz}$ bandwidth of our analyzer does not preclude significant noise contributions to (5). Integration over the entire range displayed in Fig. 5 yields $\delta f_{\text {CEO }} \approx 100 \mathrm{kHz}$ for the unstabilized laser with

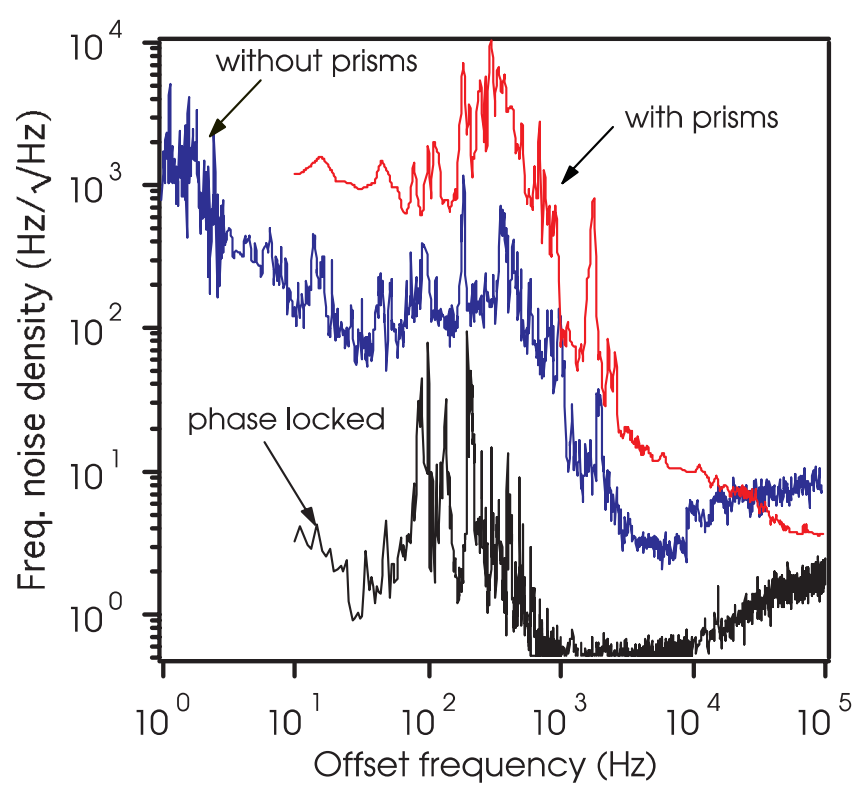

FIGURE 5 Frequency noise spectrum of the CEO frequency. Shown are three traces: unstabilized laser with intracavity prisms (top trace), laser using only chirped mirrors for dispersion compensation with (bottom trace) and without (middle trace) active stabilization. Note that the bottom trace is already very close to the detection limit for most of the range shown prisms. In the prism-less set-up, this is reduced to $0.7 \mathrm{kHz}$ or to $10 \mathrm{kHz}$, with or without active stabilization, respectively. For active stabilization a phase-lock to a reference oscillator (HP8663A) is established. The increase of noise at frequencies above $30 \mathrm{kHz}$ agrees well with the measured bandwidth of the servo loop. Using (3) one can now estimate typical pulse-to-pulse jitters of $\Delta \varphi_{\text {CEO }}$ and finds that they are negligibly small compared to $\pi$. For most applications in extreme nonlinear optics, however, it is mandatory to estimate fluctuations of $\varphi_{\text {CEO }}$ on much longer time scales than the roundtrip time. One example could be the temporal delay between firing the flash lamps of a pump laser in an amplifier and selecting a pulse with a particular CEO phase for amplification in a pulse picker. In this case, one needs to be able to predict the phase evolution of the CEO for, for example, several tens of $\mu$ s, i.e. an $f_{\text {low }}$ of a few $10 \mathrm{kHz}$. Other experiments may demand longer intervals of a stable phase of seconds or even minutes and accordingly lower values of $f_{\text {low }}$. For an estimation of the rms phase jitter, we therefore calculate from the measured noise data

$$
\begin{aligned}
\delta \varphi_{\mathrm{CEO}} & =\sqrt{2 \int_{f_{\text {low }}}^{f_{\text {high }}} \sigma_{\varphi_{\mathrm{CEO}}}^{2}(f) \mathrm{d} f} \\
& =2 \pi \sqrt{2 \int_{f_{\text {low }}}^{f_{\text {high }}}\left(\frac{\sigma_{f_{\mathrm{CEO}}}(f)}{f}\right)^{2} \mathrm{~d} f .}
\end{aligned}
$$

As the lower bound of the integration is dictated by the time that an application requires a stable CEO phase, we chose to display (6) with fixed upper bound $\left(f_{\text {high }}=100 \mathrm{kHz}\right)$ as a function of $f_{\text {low }}$. The integrated phase jitter $\delta \varphi_{\text {CEO }}\left(f_{\text {low }}\right)$ is displayed in Fig. 6 for the unstabilized lasers of Fig. 5. This plot clearly reveals the dominant contributions to the rms phase jitter as steps. For the unstabilized lasers, the most severe noise contributions are centered at $1 \mathrm{kHz}$. Integration over the entire frequency range, for example, yields a rms phase jitter $\delta \varphi_{\text {CEO }}$ of 10000 rad in the unstabilized laser with intracavity prisms. For characterizing the quality of the phaselock we switch to a different method. When deviations of the phase from the desired value are small, the phase jitter can be directly and very sensitively evaluated with an rf lock-in amplifier. For measurement of the bottom trace in Fig. 6 we utilized a Stanford Research SR844 lock-in amplifier, which provides a phase-proportional voltage at an update rate of $20 \mathrm{kHz}$ and allows us to directly evaluate $\sigma_{\varphi_{\text {CFO }}}(f)$ in (6). In particular, this method also extends the noise measurements to well below $1 \mathrm{~Hz}$. The phase noise measurements provide an independent proof of the successful phase-lock and indicate $\delta \varphi_{\mathrm{CEO}}=0.02 \mathrm{rad}$ for the frequency range from $0.01 \mathrm{~Hz}$ to $10 \mathrm{kHz}$. At the high-frequency side, these measurements can be augmented by the less reliable data derived from frequency noise density measurements, which provide an upper estimate of $\delta \varphi_{\text {CEO }} \leq 0.2 \mathrm{rad}$.

It needs to be pointed out that our phase noise characterization of the stabilized laser is fully independent of the stabilization circuitry, i.e. we do not analyze the servo loop error signal, but measure the CEO phase with an independent phase 


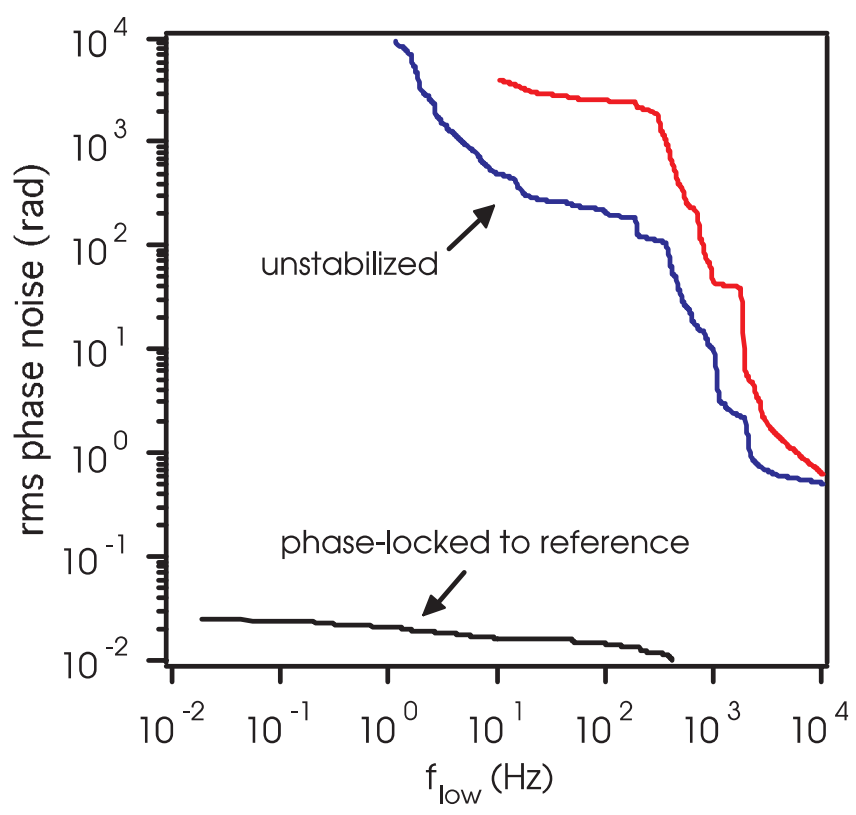

FIGURE 6 Integrated CEO phase noise spectra as a function of lower integration bound $f_{\text {low. }}$. The top two traces for the unstabilized lasers (intracavity prisms, dashed and prism-less, dotted) are deduced from the data in Fig. 5 employing (6). The bottom trace displaying the much lower phase noise of the stabilized laser (solid) is measured with an rf lock-in amplifier using the same reference oscillator as for stabilization. The upper cutoff $f_{\text {high }}$ is $100 \mathrm{kHz}$ in the top two measurements and $10 \mathrm{kHz}$ in the bottom measurement

detector. This provides proof of cycle-slip-free stabilization up to the maximum bandwidth of the rf lock-in amplifier of about $100 \mathrm{kHz}$. Nevertheless, this method would not reveal phase drifts in the optical set-up, e.g. in the interferometer or the fiber itself. Similar measurements of the CEO phase noise were reported [18]. These authors also established a phaselock, but did not further characterize residual phase jitter for low frequencies. They estimate $\delta \varphi_{\text {CEO }} \leq 0.3$ rad for frequencies above $100 \mathrm{~Hz}$ from a measurement of pulse-energy fluctuations. This method, however, presupposes a strict correlation of power and CEO frequency in the laser. Recently, stabilization of the CEO phase of two independent lasers was also reported [19]. Those authors reported loss of interferencefringe contrast between the two lasers, i.e. a $\delta \varphi_{\mathrm{CEO}}$ on the order of $\pi$, at observation times below $20 \mathrm{~ms}$ corresponding to $f_{\text {low }}=50 \mathrm{~Hz}$. Apart from methodical differences in the measurements, these examples show that so far negligible $\delta \varphi_{\text {CEO }} \ll \pi$ was not reported for extended observation times of seconds or more.

The difficulties experienced in reaching a long-term stabilization of the CEO phase and the strong difference between the noise in lasers with intracavity prisms and prismless lasers clearly demonstrate the necessity of reaching a deeper understanding of the underlying physical processes causing fluctuations of the carrier-envelope offset. This will be addressed in Sect. 4.

\section{The physical origin of carrier-envelope fluctuations}

Several coupling mechanisms between carrierenvelope-offset phase and intracavity power have been pro- posed. One of the main applications of such a mechanism is the ability to control $f_{\text {CEO }}$ externally by adjusting the pump power. $\mathrm{Xu}$ et al. observed a power-dependent shift of the mode-locked spectrum [7]. The influence of this spectral shift on the carrier frequency of the pulse was used to explain the power-dependent change of the carrier-envelope frequency also observed in these experiments. Later, this amplitude-tophase conversion mechanism was explained by self-phase modulation [20]. An additional coupling mechanism due to beam-pointing variations in the prism compressor was also suspected by Morgner et al. [14]. However, with the excessive noise of the laser used in this investigation, the authors were unable to provide any experimental evidence for this coupling mechanism.

To explore amplitude-to-phase coupling mechanisms in our laser, we introduce an acousto-optic modulator between the pump laser and the Ti:sapphire oscillator. The experiments described in this section have been carried out with the laser with intracavity prisms. The AOM deflects a small portion of the pump power into the first diffraction order while the zeroth order of the AOM is used to pump the Ti:sapphire laser. The drive power to the modulator is periodically modulated with a reference signal, which is also used for phase-synchronous detection of the resulting modulation of $f_{\mathrm{CEO}}$ and beam pointing, see Fig. 3. Thermal and environmental contributions to these quantities have been minimized by use of modulation frequencies of several $\mathrm{kHz}$. The CEO frequency is measured as described in Sect. 3, and the beam position is monitored with a position-sensitive detector (Sitek 1L2, 5SP) using one of the residual reflections off the intracavity Brewster-prism surfaces.

Using a lock-in technique, we carefully measured the change of the $\mathrm{CEO}$ frequency induced by a modulation of the intracavity peak power. In our experiments we also checked for changes in pulse duration. We find that a change of intracavity pulse energy is only accompanied by a relatively small change of pulse duration in the laser. Using several different modulation frequencies, we measure that the CEO frequency changes by $5 \times 10^{-4} \mathrm{~Hz}$ per $\mathrm{W} / \mathrm{cm}^{2}$ change of intracavity intensity. Similarly, we find that modulation of the intracavity power induces a beam displacement on the order of $10^{-15} \mathrm{~m}$ per $\mathrm{W} / \mathrm{cm}^{2}$ change of intensity inside the laser crystal. The beam movement is measured at the location shown in Fig. 2. Note that a $1 \%$ modulation of the intracavity intensity translates into a beam movement of $4.5 \mu \mathrm{m}$, corresponding to a pointing variation of several microradians.

It is obvious from (1) that only fluctuations of the linear dispersion $\mathrm{d} n / \mathrm{d} \omega$ have a strong effect on the CEO frequency of a laser cavity. Cavity-length fluctuations, however, may contribute only by affecting $f_{\text {rep }}$ in (3). Typically, we observe fluctuations of the repetition rate of less than $100 \mathrm{~Hz}$ per second in the unstabilized laser. This cannot account for the $\mathrm{MHz}$ sweeps of the CEO frequency observed in some of the early measurements without proper environmental shielding. In comparison to the problem of stabilizing the frequency of a continuous single-mode laser, the contribution of acoustic and thermal length variations via displacement of the cavity mirrors is clearly negligible. This leaves dispersion variations of the intracavity elements as the major mechanism causing $\mathrm{CEO}$ frequency fluctuations. These variations can be ther- 
mally or acoustically induced or they can be caused by nonlinear refraction [20]. For our laser cavity, we estimate a change $\Delta f_{\text {CEO }} \approx 1.4 \mathrm{MHz}$ for a $1-\mathrm{K}$ temperature change of the laser crystal [21]. Similarly, we calculate $\Delta f_{\mathrm{CEO}} \approx 20 \mathrm{kHz}$ for an air-pressure variation of $1 \mathrm{~Pa}$ [22]. These mechanisms can be easily shielded or will only cause a slow drift of the CEO frequency. Therefore we concentrate on nonlinear-optical refractive mechanisms in the following.

Let us first explore a direct change of the refractive index via the Kerr effect. According to (1), only the linear dispersion of self-refraction plays a role. Typically, this effect is treated in the time domain by inclusion of an additional time-derivative term in the nonlinear Schrödinger equation [23]. This term accounts for the linear dispersion of the Kerr nonlinearity. The accompanying reshaping of the pulse is usually referred to as self-steepening. For our purposes we use the formalism developed in [24] for a direct estimation of the linear dispersion of the Kerr effect in the frequency domain. Based on this analysis, the nonlinear effect on the average group-phase offset of a pulse is calculated. We estimate the effective groupphase offset coefficient of sapphire at $800-\mathrm{nm}$ wavelength as $\omega \partial n_{2} / \partial \omega=8 \times 10^{-17} \mathrm{~cm}^{2} / \mathrm{W}$. This formalism also estimates self-refraction as $n_{2}=3 \times 10^{-16} \mathrm{~cm}^{2} / \mathrm{W}$, which agrees very well with experimental data [25]. The computed groupphase offset leads to a theoretical value of $\partial f_{\mathrm{CEO}} / \partial I=4 \times$ $10^{-5} \mathrm{~Hz}$ per $\mathrm{W} / \mathrm{cm}^{2}$ for our laser, i.e. about one order of magnitude less than observed in the experiments.

Beam-pointing variations may be induced by self-refraction at the interface between the Brewster-cut Ti:sapphire crystal and air as illustrated in Fig. 7. If the index inside the crystal changes, Snell's law demands a change of beam angles. In principle, a change of the index of refraction of the laser material can induce both a change of the outside and the inside beam angles $\left(\vartheta_{\text {ext }}\right.$ and $\vartheta_{\text {int }}$, respectively). However, the pump-beam direction is not affected by the Kerr-induced refraction change, which only lasts for a few femtoseconds within the 10-ns roundtrip time of the laser. The overlap between pump mode and laser mode therefore favors a change of the outside angle. Now assuming that $\vartheta_{\text {int }}$ is fixed, one can calculate a coarse estimate for the magnitude of beampointing movements as $\partial \vartheta_{\text {ext }} / \partial I=3 \times 10^{-16} \mathrm{rad} \mathrm{cm}^{2} / \mathrm{W}$. It is clear that the calculated value may only serve as an upper estimate for the nonlinear beam-steering effect, but this value is compatible with the measured beam-position movement.

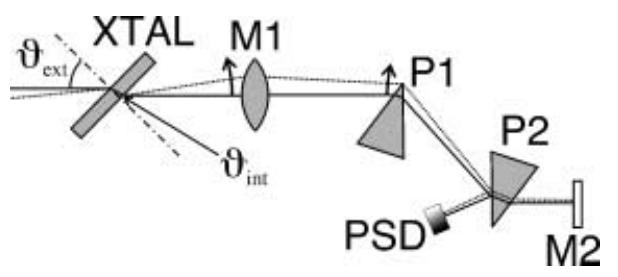

FIGURE 7 Suggested beam-pointing variations induced by nonlinear refraction in the gain crystal (XTAL). The nonlinear beam steering induces a differential material insertion at the two intracavity prisms P1 and P2, which leads to changes of the group-phase offset. M1 is an intracavity folding mirror, M2 an end mirror or output coupler, PSD the position-sensitive detector. Note that the experimentally detected beam movements are small in comparison to the numerical aperture of the beams. The pump acts as a geometric filter and constantly forces the laser mode to maximum overlap with the pumped volume inside the laser crystal
The change of the beam orientation inside the intracavity prism compressor is accompanied by a change of the group-phase offset. For the fused-silica Brewster-prism sequence with $30-\mathrm{cm}$ apex separation used in our laser, we calculate a beam-pointing sensitivity of the CEO frequency $\partial f_{\text {CEO }} / \partial \vartheta=2.5 \times 10^{12} \mathrm{~Hz} / \mathrm{rad}$. While this value has been derived from a full analysis of the spectral phase of the prism compressor [26], the main effect can be easily understood from a change of the effective material insertion in the prism compressor. A change of beam direction, for example, reduces material insertion at the first prism and increases insertion at the second prism. These effects only cancel out if the pivot point of the beam movement is right at the midpoint between the two prisms (compare Figs. 7 and 8). Finally, we combine the calculations of nonlinear beam steering and the pointing sensitivity to yield an estimate of $\partial f_{\text {CEO }} / \partial I=$ $7 \times 10^{-4} \mathrm{~Hz} \mathrm{~cm}^{2} / \mathrm{W}$. This number agrees well with the measured value of $5 \times 10^{-4} \mathrm{~Hz} \mathrm{~cm}^{2} / \mathrm{W}$.

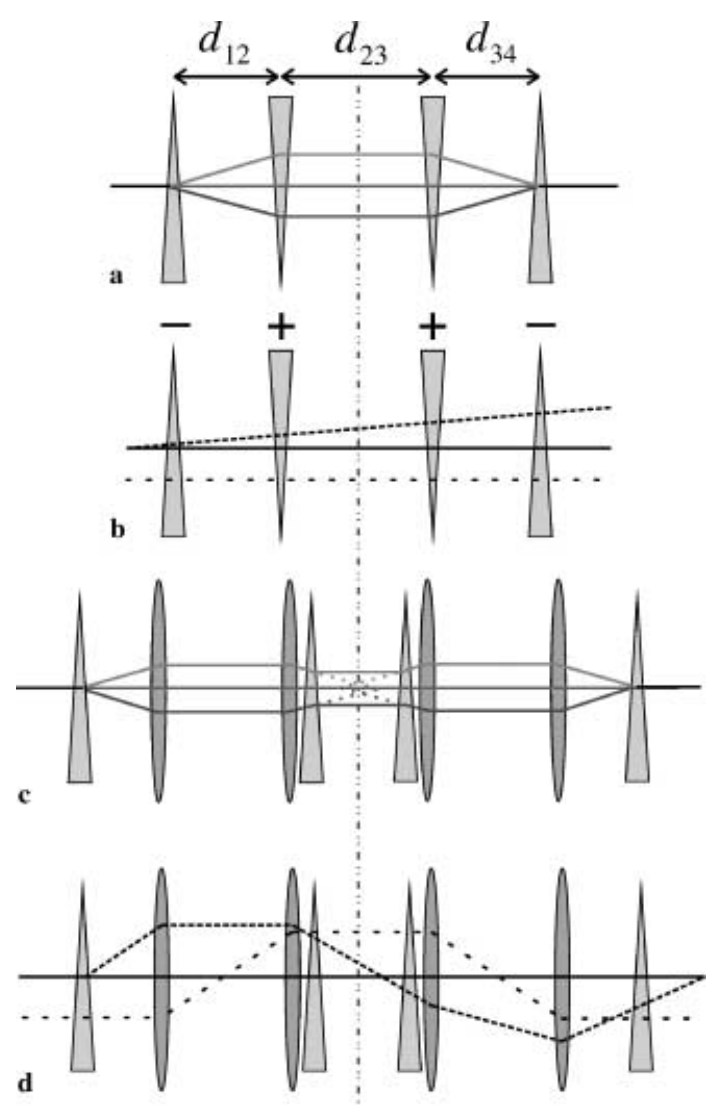

FIGURE 8 Illustration of the beam-pointing effects in sequences of angular dispersive elements. The principal center-wavelength ray is drawn as a straight line. The changes of material insertion in this figure can be interpreted as a change of group-phase offset in a grating sequence. a Compressor sequence with three rays of different wavelengths, as designed. This set-up generates negative second-order dispersion as the outer rays experience an increased optical path length compared to the principal ray. b Translated (dashed line) and rotated (dotted line) principal ray in a stretcher. Net material insertion remains constant when $d_{12}=d_{34}$, i.e. the GPO is not affected. c Stretcher sequence with three rays of different wavelengths, as designed. The telescopes invert the phase-front curvature compared to a, yielding positive second-order dispersion. d Translational (dashed) and rotational (dotted) movement of the principal ray. Beam rotation influences material insertion, resulting in a net effect on the GPO of the stretcher sequence 
Similar mechanisms based on thermally induced index changes and their dispersion may also be present but have not been studied here. It is expected that they play a strong role for frequencies below $1 \mathrm{kHz}$. All amplitude-to-phase conversion mechanisms work both ways: first they allow the control of the CEO frequency of a cavity by simply modulating intracavity peak power. Second, stabilization of the CEO frequency will also strongly suppress pulse-energy fluctuations via the described coupling mechanisms. This inverse coupling explains the reduction of pulse-energy noise found in a CEO-stabilized laser. It should be pointed out that our explanation of the coupling mechanism via self-steepening is consistent with the spectral shift observed by Xu et al. [7]. Self-steepening is known to cause a spectral asymmetry of the nonlinear refractive broadening [27], even though it may be difficult to give a quantitative estimate for the carrier-envelope-offset change expected for a certain spectral shift.

\section{Implications for maintaining the CEO stabilization in chirped-pulse amplifiers}

As outlined in Sect. 4, an understanding of the mechanisms behind the CEO fluctuations allows for the design of oscillators with the least possible coupling of amplitude to phase. For many applications of CEO-stabilized sources, it is necessary to amplify pulses to the $\mu \mathrm{J}$ or $\mathrm{mJ}$ level and maintain the stabilized phase. In the following, we will analyze how the mechanisms that have been found important in the oscillator influence the amplification of pulses.

Let us start with the most severe effect in the oscillator, nonlinear beam steering in combination with the angular sensitivity of geometrical dispersion schemes. External femtosecond amplification schemes nearly exclusively rely on chirped pulse amplification (CPA, [28]). This requires the pulse to be stretched before and recompressed after amplification. For this purpose, grating sequences based on the Treacy compressor have found widespread use [29]. In the standard Treacy grating compressor the group-phase offset induced by the grating phase [30] at the second grating is a particular concern. Beam-pointing variations leading to a displacement by a single-groove spacing at a grating translate into a $2 \pi$ change of group-phase offset. This effect is equivalent to the additional material insertion in prism sequences induced by beam-pointing variations. However, grating sequences are much more sensitive to a change of beam direction. For example, a typical grating distance of a few tens of $\mathrm{cm}$ and a grating period of $1000-2000$ grooves $/ \mathrm{mm}$ restricts tolerable beam movements to much less than a microradian. To analyze the net effect in complex stretcher and compressor sequences, we restrict ourselves to the more illustrative picture of prisms in Fig. 8. Equivalently, the change of material path in this figure can always be interpreted as a change of GPO in a grating sequence. In Fig. 8a and c, a compressor and a stretcher sequence are schematically drawn with the principal center-wavelength ray as a straight line. Angular dispersion in the first prism leads to an increased path length for wavelengths shorter or longer than the principal ray wavelength. This parabolic phase front gives the dominant contribution to the negative second-order dispersion of such an arrangement. Angular movement of the principal ray (dotted line in Fig. 8b) now changes the group-phase offset at each of the four prisms. The material/grating GPO is reduced at prisms 1 and 4 and increased at prisms 2 and 3. From inspection of Fig. 8b, it becomes clear that these effects cancel out if $d_{12}$ is chosen to be identical to $d_{34}$, with an arbitrary choice of $d_{23}$. A translational movement (dashed line) does not play a role. The typical compressor arrangement uses only two prisms in double-pass, which automatically ensures $d_{12}=d_{34}$. In this case, compressor grating sequences are not expected to be susceptible to beam-movement-induced effects on the CEO phase. Note that this treatment does not automatically hold for intracavity prism compressors but also depends on the exact cavity configuration of the laser. Also, the sensitivity towards beam pointing changes dramatically as soon as imaging elements are introduced into the prism/grating sequences.

A typical stretcher arrangement is inspected in Fig. 8c and $\mathrm{d}$. In a stretcher, the diverging bundle of rays is converted into a converging one by means of a $1: 1$ telescope. This inverts the phase-front curvature of the beam and allows one to generate positive second-order dispersion exactly canceling out the dispersion of an equivalent compressor arrangement. In such an arrangement, the direction of the beam is changed at each of the telescopes when the beam is moved out of centration (see Fig. 8d). This applies to both a translational (dashed) and a rotational (dotted) movement of the beam. If the pivot point of the beam movement is exactly known, $d_{23}$ could be chosen such that the beam always crosses the principal design ray at the location of the rear mirror (dash-dotted line in Fig. 8). Knowledge of the geometry of beam-pointing variations may then at least allow a reduction of the effect. As several different mechanisms may contribute, we expect that beam-pointing variations still have to be kept in the few$\mu$ rad range to avoid degradation of a stabilized CEO phase in a grating stretcher.

The second concern derived from the analysis of the oscillator is amplitude-to-phase conversion via self-steepening. As in the oscillator, this effect seems to be much less of a problem because typically amplifiers are designed such that the $B$-integral, i.e. the cumulated nonlinear phase, is smaller than $2 \pi$. Using the numbers derived in Sect. 4 , the total selfsteepening contribution to the $\mathrm{CEO}$ phase is then expected to be smaller than $0.5 \pi$. This demands a $6 \%$ shot-to-shot energy stability of the amplifier to keep effects on the CEO phase below $0.1 \mathrm{rad}$.

Amplitude-to-phase coupling is expected to be a much more severe problem when additional external compression [31] is employed. Because of the complex nonlinear mechanism in femtosecond continuum generation, it is very difficult to derive a reliable estimate of the severity of amplitude-to-phase coupling. We estimate that these effects are at least one order of magnitude stronger than the intrinsic nonlinearities of the amplifier itself. This would exclude amplifier systems with strong (>10\%) shot-to-shot energy fluctuations, but CEO stabilization might still be manageable with amplifiers with state-of-the-art stability.

With the much lower repetition rates of amplifier systems, environmental influences deserve a closer inspection. With the numbers derived in Sect. 4 and assuming a typical path length of $10 \mathrm{~cm}$ of Ti:sapphire in the amplifier, a tempera- 
ture change of $1 \mathrm{~K}$ would effect a 1.9-rad change of the CEO phase. Similarly, an air-pressure variation of $1 \mathrm{~Pa}$ would induce about a 1-mrad CEO phase change in a 10-m path length.

In summary, stabilization of the CEO phase in a chirpedpulse amplifier is a very challenging problem, in particular because of the severity of beam-pointing effects. These effects do not play a role if only plane gratings are considered, as in the compressor. However, as soon as gratings and imaging elements are combined, a severe coupling mechanism between beam pointing and CEO phase is introduced. This explains the observed effects in the oscillator itself, and it disfavors the use of stretcher grating arrangements. Moreover, we also expect $4 f$ shapers for adaptive pulse compression [32] to experience similar problems. The most viable option for avoiding detrimental effects in the stretcher seems to be the use of a bulk material for this purpose, as described in [33]. This solution may not allow for the shortest possible pulse duration but it is insensitive to beam-pointing-induced fluctuations of the CEO phase. As the pulses have to be compressed in any case to reach the regime of extreme nonlinear optics, the limited pulse duration does not appear to be a major problem. Using a heavy-flint glass stretcher, thermal influences on the CEO phase will require additional care. Thermal changes in the amplifier and nonlinear optical effects in a subsequent hollow-fiber compressor may well add up. At this point, the only practical solution for phase-sensitive experiments with amplified pulses seems to be monitoring of the CEO phase [34-36] together with binning of the experimental results.

6

\section{Conclusions}

We have identified several mechanisms that cause a conversion of amplitude fluctuations into a change of the cavity group-phase offset. The most serious concern, both in oscillators and in amplified systems, is a translation of beampointing variation into a phase change, which is a particular problem of geometrical dispersion-compensation schemes. In principle, this effect can be avoided by suitable choice of the dispersion-compensating elements. Our results strongly suggest the use of prism-less dispersion compensation in oscillators and bulk stretchers for chirped pulse amplification if the carrier-envelope phase is a concern. Nevertheless, nonlinear effects, such as self-steepening, and environmental effects, like air-pressure variations and temperature changes, cannot be totally avoided.

From the discussion carried out so far, the impression may arise that amplitude-to-phase coupling effects have to be avoided at any expense. However, the same effect that converts amplitude fluctuations into phase noise can also be used to control carrier-envelope fluctuations. Most suitably, this is done by a fast electro-optic or acousto-optic modulator between pump laser and oscillator. Here we utilized this scheme to establish a phase-lock of the CEO frequency to a reference oscillator. A careful analysis revealed a residual jitter of the CEO phase as low as $20 \mathrm{mrad}$ in a $0.01-\mathrm{Hz}$ to $10-\mathrm{kHz}$ bandwidth. If amplitude noise and phase noise are strictly correlated, suppression of the CEO noise may also serve to reduce amplitude noise.
ACKNOWLEDGEMENTS The authors thank R.S. Windeler, Lucent Technologies, for providing us with the microstructure fiber. We gratefully acknowledge the help of B. Lipphardt, PTB Braunschweig, in setting up the phase-lock and the noise-measurement equipment of Sect. 3. We thank Lukas Gallmann, ETH Zürich, for experimental assistance. This research is financially supported by ETH Zürich under Grant No. 03159/41.

\section{REFERENCES}

1 G. Steinmeyer, D.H. Sutter, L. Gallmann, N. Matuschek, U. Keller: Science 286, 1507 (1999)

2 P. Dietrich, F. Krausz, P.B. Corkum: Opt. Lett. 25, 16 (2000)

3 A. de Bohan, P. Antoine, D.B. Milosevic, B. Piraux: Phys. Rev. Lett. 81, 1837 (1998)

4 M. Drescher, M. Hentschel, R. Kienberger, G. Tempea, C. Spielmann, G.A. Reider, P.B. Corkum, F. Krausz: Science 291, 1923 (2001)

5 M. Lenzner, M. Schnürer, C. Spielmann, F. Krausz: IEICE Trans. Electron. E81C, 112 (1998)

6 D.H. Sutter, G. Steinmeyer, L. Gallmann, N. Matuschek, F. MorierGenoud, U. Keller, V. Scheuer, G. Angelow, T. Tschudi: Opt. Lett. 24, 631 (1999)

7 L. Xu, C. Spielmann, A. Poppe, T. Brabec, F. Krausz, T.W. Hänsch: Opt. Lett. 21, 2008 (1996)

8 H.R. Telle, G. Steinmeyer, A.E. Dunlop, J. Stenger, D.H. Sutter, U. Keller: Appl. Phys. B 69, 327 (1999)

9 T. Udem, J. Reichert, R. Holzwarth, T.W. Hänsch: Opt. Lett. 24, 881 (1999)

10 D.J. Jones, S.A. Diddams, J.K. Ranka, A. Stentz, R.S. Windeler, J.L. Hall, S.T. Cundiff: Science 288, 635 (2000)

11 A. Apolonski, A. Poppe, G. Tempea, C. Spielmann, T. Udem, R. Holzwarth, T.W. Hänsch, F. Krausz: Phys. Rev. Lett. 85, 740 (2000)

12 J. Stenger, T. Binnewies, G. Wilpers, F. Riehle, H.R. Telle, J.K. Ranka, R.S. Windeler, A.J. Stentz: Phys. Rev. A 63, 021802 (2001)

13 J.K. Ranka, R.S. Windeler, A.J. Stentz: Opt. Lett. 25, 25 (2000)

14 U. Morgner, R. Ell, G. Metzler, T.R. Schibli, F.X. Kärtner, J.G. Fujimoto, H.A. Haus, E.P. Ippen: Phys. Rev. Lett. 86, 5462 (2001)

15 R. Szipöcs, K. Ferencz, C. Spielmann, F. Krausz: Opt. Lett. 19, 201 (1994)

16 F.X. Kärtner, N. Matuschek, T. Schibli, U. Keller, H.A. Haus, C. Heine, R. Morf, V. Scheuer, M. Tilsch, T. Tschudi: Opt. Lett. 22, 831 (1997)

17 U. Keller, K.D. Li, M.J.W. Rodwell, D.M. Bloom: IEEE J. Quantum Electron. QE-25, 280 (1989)

18 A. Poppe, R. Holzwarth, A. Apolonski, G. Tempea, C. Spielmann, T.W. Hänsch, F. Krausz: Appl. Phys. B 72, 373 (2000)

19 R.K. Shelton, L.-S. Ma, H.C. Kapteyn, M.M. Murnane, J.L. Hall, J. Ye: Science 293, 1296 (2001)

20 J. Stenger, H.R. Telle: Opt. Lett. 25, 1553 (2000)

21 M.E. Thomas, S.K. Andersson, R.M. Sova, R.I. Joseph: Infrared Phys. Technol. 39, 235 (1998)

22 R. Muijlwijk: Metrologia 25, 189 (1988)

23 G.P. Agrawal: Nonlinear Fiber Optics (Academic, New York 1989)

24 M. Sheik-Bahae, D.C. Hutchings, D.J. Hagan, E.W. Van Stryland: IEEE J. Quantum Electron. QE-27, 1296 (1991)

25 R. DeSalvo, A.A. Said, D.J. Hagan, E.W. Van Stryland, M. Sheik-Bahae: IEEE J. Quantum Electron. QE-32, 1324 (1996)

26 R.E. Sherriff: J. Opt. Soc. Am. B 15, 1224 (1998)

27 T.K. Gustafson, J.P. Taran, H.A. Haus, J.R. Lifsitz, P.L. Kelley: Phys. Rev. 177, 306 (1969)

28 D. Strickland, G. Mourou: Opt. Commun. 56, 219 (1985)

29 E.B. Treacy: IEEE J. Quantum Electron. QE-5, 454 (1969)

30 H.A. Haus: Waves and Fields in Optoelectronics (Prentice Hall, Englewood Cliffs, NJ 1984)

31 M. Nisoli, S. De Silvestri, O. Svelto, R. Szipöcs, K. Ferenz, C. Spielmann, S. Sartania, F. Krausz: Opt. Lett. 22, 522 (1997)

32 A.M. Weiner: Rev. Sci. Instrum. 71, 1929 (2000)

33 M. Hentschel, Z. Cheng, F. Krausz, C. Spielmann: Appl. Phys. B 70, S161 (2000)

34 M. Mehendale, S.A. Mitchell, J.P. Likforman, D.M. Villeneuve, P.B. Corkum: Opt. Lett. 25, 1672 (2000)

35 M. Kakehata, H. Takada, Y. Kobayashi, K. Torizuka, Y. Fujihira, T. Homma, H. Takahashi: Opt. Lett. 26, 1436 (2001)

36 M. Kakehata, Y. Fujihira, H. Takada, Y. Kobayashi, K. Torizuka, T. Homma, H. Takahashi: Appl. Phys. B, Proc. Ultrafast Opt. Conf., submitted 